Revista de Matemática: Teoría y Aplicaciones 2(2): 67-73 (1995)

\title{
LA EXPANSIÓN ASINTÓTICA DE NÚCLEOS DISTRIBUCIONAL
}

\author{
Carlos Manuel Ulate Ramírez ${ }^{1}$
}

\begin{abstract}
Resumen
Este artículo utiliza la técnica de expansión asintótica parcial aplicada a distribuciones de orden finito, con el fin de obtener la expansión distribucional de núcleos de la forma $f(\lambda \mathbf{x}, \mathbf{y})$, cuando $\lambda \rightarrow+\infty$ y $\mathbf{x}=\mathbf{y}$. Se introducen condiciones sobre $f$, que nos permite obtener la expansión de ciertas integrales de la forma $\int_{\mathbb{R}^{n}} f(\lambda \mathbf{x}, \mathbf{x}) \phi(\mathbf{x}) d \mathbf{x}$ cuando $\lambda \rightarrow+\infty$; y con ello generalizar los resultados obtenidos en [10, 11].
\end{abstract}

\section{Introducción}

En el estudio de operadores singulares $[1,2]$ a menudo se necesita conocer la expansión de integrales de la forma

$$
\int_{\mathbb{R}^{n}} f(\lambda \mathbf{x}, \mathbf{x}) \phi(\mathbf{x}) d \mathbf{x}, \quad \lambda \rightarrow+\infty .
$$

Recientemente Sellier $[6,7]$ utiliza el concepto de parte finita en el sentido de Hadamard $n \geq 2$, específicamente en el sentido de valor principal, con el fin de estudiar ciertas funciones singulares del tipo (1.1).

El siguiente análisis se basa en la expansión asintótica de momentos parcial $[3,4]$

$$
f(\lambda \mathbf{x}, \mathbf{y}) \sim \sum_{|\mathbf{k}|=0}^{\infty} \frac{(-1)^{|\mathbf{k}|} \mu_{\mathbf{k}}(\mathbf{y}) \mathbf{D}^{\mathbf{k}} \delta(\mathbf{x})}{\mathbf{k} ! \lambda^{|\mathbf{k}|+N}}, \text { cuando } \lambda \rightarrow+\infty
$$

valida para algunos espacios de distribuciones sobre $\mathbb{R}^{n} \times V$, donde $V$ es una variedad.

Los momentos $\mu_{\mathbf{k}}(\mathbf{y})=\left\langle f(\mathbf{x}, \mathbf{y}), \mathbf{x}^{\mathbf{k}}\right\rangle, \mathbf{k} \in I^{n}$, son funciones que dependen de $\mathbf{y}$.

Introduciendo los espacios de funciones prueba de orden finito y las distribuciones de orden finito sobre $\mathbb{R}^{n}$, obtendremos la versión distribucional de la expansión asintótica para núcleos como los considerados en (1.1).

Observe que en general no podemos poner $\mathbf{x}=\mathbf{y}$ en la expansión (1.2), por ejemplo la expansión del núcleo de Fourier [3, 4]

$$
e^{i \lambda \mathbf{x} \cdot \mathbf{y}} \sim(2 \pi)^{n} \sum_{|\mathbf{k}|=0}^{\infty} \frac{i^{|\mathbf{k}|} \mathbf{D}^{\mathbf{k}} \delta(\mathbf{x}) \mathbf{D}^{\mathbf{k}} \delta(\mathbf{y})}{\mathbf{k} ! \lambda^{|\mathbf{k}|+n}}, \text { cuando } \lambda \rightarrow+\infty
$$

\footnotetext{
${ }^{1}$ Sede de Occidente, Universidad de Costa Rica
} 
la cual es válida por ejemplo en $\mathcal{E}\left(\mathbb{R}^{n}\right) \widehat{\otimes}_{\pi} \mathcal{F}\left(\mathcal{E}^{\prime}\left(\mathbb{R}^{n}\right)\right)$. Si hacemos $\mathbf{x}=\mathbf{y}$ se obtiene la expresión indefinida $[9,11]\left(\mathbf{D}^{\mathbf{k}} \delta(\mathbf{x})\right)^{2}$.

En lo que sigue de este trabajo usamos la notación usual de multi-índices, así por ejemplo si $\mathbf{k} \in I N^{n}$, entonces $\mathbf{k} !=k_{1} ! \ldots k_{n} !, \quad|\mathbf{k}|=k_{1}+\ldots+k_{n}, \quad \mathbf{D}^{\mathbf{k}}=\frac{\partial^{k_{1}}}{\partial x_{1}^{k_{1}}} \ldots \frac{\partial^{k_{n}}}{\partial x_{n}^{k_{n}}}$.

De este modo los correspondientes momentos en (1.2), están definidos por $\mu_{\mathbf{k}}\left(y_{1}, \ldots, y_{n}\right)=\left\langle f\left(x_{1}, \ldots, x_{n}, y_{1}, \ldots, y_{n}\right), x_{1}^{k_{1}} \cdot \ldots \cdot x_{n}^{k_{n}}\right\rangle, \mathbf{k} \in \mathbb{I}^{n}$.

\section{Espacio de distribuciones de orden finito}

Introducimos ahora los espacios de funciones prueba y distribuciones que se utilizan en este análisis; $\mathcal{E}^{m}\left(\mathbb{R}^{n}\right)$, el espacio de todas las funciones continuamente diferenciables de orden $m(m \in I N)$ sobre $\mathbb{R}^{n}$. El espacio dual $\mathcal{E}^{\prime m}\left(\mathbb{R}^{n}\right)$, el espacio de distribuciones de soporte compacto de orden finito $m$.

$\mathcal{D}^{m}\left(\mathbb{R}^{n}\right)$, el espacio de las funciones continuamente diferenciables hasta de el orden $m$ con soporte compacto cotenido en $\mathbb{R}^{n}$, esto es las funciones $\phi$ para las cuales $\mathbf{D}^{p} \phi(x)$ existe y es continua para $|p| \leq m$ y cuyo soporte compacto esta contenido en $\mathbb{R}^{n}$. El espacio $\mathcal{D}^{\prime m}\left(\mathbb{R}^{n}\right)$, espacio de distribuciones de orden finito.

$\mathcal{S}^{m}(\mathbb{R})$, el espacio de las funciones cuyas derivadas de orden menor o igual a $m$ son rápidamente decrecientes, esto es $\lim _{\mathbf{x} \rightarrow \infty} \mathbf{x}^{\mathbf{k}} \mathbf{D}^{\mathbf{j}} \phi(\mathbf{x})=0$ para cada par de multi-índices $\mathbf{k}, \mathbf{j} \in I N^{n}, \quad|\mathbf{j}| \leq m$.

El espacio dual $\mathcal{S}^{\prime m}\left(\mathbb{R}^{n}\right)$ es el espacio de las distribuciones temperadas.

El espacio $\mathcal{O}_{\gamma}^{m}\left(\mathbb{R}^{n}\right), \gamma \in \mathbb{R}$ consiste de las funciones suaves tales que $\mathbf{D}^{\mathbf{k}} \phi(\mathbf{x})=$ $\mathcal{O}\left(|\mathbf{x}|^{\gamma}\right)$, cuando $\mathbf{x} \rightarrow \infty$ para cada $\mathbf{k} \in \mathbb{N}^{n}, \quad|\mathbf{k}| \leq m$. El espacio $\mathcal{O}_{C}^{m}\left(\mathbb{R}^{n}\right)$ es el límite inductivo $\lim _{\rightarrow} \mathcal{O}_{\gamma}^{m}\left(\mathbb{R}^{n}\right)$ cuando $\gamma \rightarrow \infty$. El espacio $\mathcal{K}^{m}\left(\mathbb{R}^{n}\right)$ consiste en las funciones suaves tal que $\mathbf{D}^{\mathbf{k}} \phi(\mathbf{x})=O\left(|\mathbf{x}|^{\gamma-|\mathbf{k}|}\right)$ para algún $\gamma$ y todo $\mathbf{k} \in \mathbb{I}^{n}, \quad|\mathbf{k}| \leq m$. El espacio $\mathcal{O}_{M}\left(\mathbb{R}^{n}\right)$ de funciones suaves, tal que $\mathbf{D}^{\mathbf{k}} \phi(\mathbf{x})=O\left(|\mathbf{x}|^{\gamma_{\mathbf{k}}}\right)$ para alguna constante $\gamma_{\mathbf{k}}, \mathbf{k} \in I^{n}$.

El espacio $\mathcal{P}^{m}\left(\mathbb{R}^{n}\right)$ de las funciones suaves tales que $\mathbf{D}^{\mathbf{k}} \phi(\mathbf{x})=O\left(e^{\gamma|\mathbf{x}|}\right)$ as $\mathbf{x} \rightarrow \infty$ para cada $\gamma>0$, y $\mathbf{k} \in \mathbb{I N}^{n},|\mathbf{k}| \leq m$.

Para un estudio detallado de estos espacios y sus correspondientes duales, consultar $[3,4,5]$

En lo que sigue $\mathcal{A}^{\prime m}$, representa alguno de los espacios $\mathcal{A}=\mathcal{D}, \mathcal{E}, \mathcal{O}_{C}, \mathcal{K}, \mathcal{P}$ o $\mathcal{S}$, dotado de la topología fuerte $\beta\left(\mathcal{A}^{\prime m}, \mathcal{A}^{m}\right)$; y lo denotamos por $\mathcal{A}_{\beta}^{\prime m}$.

\section{Funciones bilineales continuas}

Sea $\mathcal{A}=\mathcal{A}\left(\mathbb{R}^{n}\right)$ un espacio de funciones prueba sobre $\mathbb{R}^{n}$ y $\mathcal{A}^{\prime}$ el correspondiente espacio de distribuciones, en general la función $\Phi: \mathcal{A}^{\prime} \times \mathcal{B} \rightarrow \mathcal{A}^{\prime}$, definida por $\Phi(T, \alpha):=\alpha T$, donde $\mathcal{B}$ es el espacio de multiplicadores [11] de $\mathcal{A}$, no es continua. Por ejemplo la función $\mathcal{D}^{\prime} \times \mathcal{E} \rightarrow \mathcal{D}$ no es continua [5].

La función $\Phi$ induce un operador [11] $\Delta: \mathcal{A}^{\prime} \times \mathcal{B} \rightarrow \mathcal{A}^{\prime}$ tal que $\Delta(T \otimes \alpha)=\alpha T$ de modo que si $\sigma(x, y)=\sum_{k=1}^{m} \alpha_{k}(\mathbf{x}) T_{k}(\mathbf{y})$, entonces $(\Delta \sigma)(\mathbf{x})=\sigma(\mathbf{x}, \mathbf{x})$. 
Si $\Phi$ es continua, entonces $\Delta$ se extiende a un operador continuo de $\mathcal{A}^{\prime} \widehat{\otimes}_{\pi} \mathcal{B} \rightarrow \mathcal{A}^{\prime}$, donde $\widehat{\otimes}_{\pi}$ significa la complexión del espacio $\mathcal{A}^{\prime} \otimes_{\pi} \mathcal{B}$ provisto de la $\pi$-topología $[3,4,8]$ o topología proyectiva.

La siguiente proposición nos garantiza cuando $\Phi$ es continua [5, pg. 362].

\section{Proposición 3.1:}

Sea $m \in I N$ entonces la función

$$
\Phi: \mathcal{D}_{\beta}^{\prime m}\left(\mathbb{R}^{n}\right) \times \mathcal{E}^{m}\left(\mathbb{R}^{n}\right) \rightarrow \mathcal{D}_{\beta}^{\prime m}\left(\mathbb{R}^{n}\right)
$$

definida por $\Phi(T, \alpha):=\alpha T$ es bilineal y continua.

Al ser la función (3.1) continua se sigue que el operador $\Delta: \mathcal{D}_{\beta}^{\prime m}\left(\mathbb{R}^{n}\right) \widehat{\otimes}_{\pi} \mathcal{E}^{m}\left(\mathbb{R}^{n}\right) \rightarrow$ $\mathcal{D}_{\beta}^{\prime m}\left(\mathbb{R}^{n}\right)$, definido por $(\Delta \sigma)(\mathbf{x})=\sigma(\mathbf{x}, \mathbf{x})$ es continuo.

Como $\mathcal{E}^{\prime m} \hookrightarrow \mathcal{D}^{\prime m}$ y $\mathcal{E} \hookrightarrow \mathcal{E}^{\prime m}$, se tiene que $\Phi: \mathcal{E}^{\prime m} \times \mathcal{E} \rightarrow \mathcal{D}^{\prime m}$ es continua y por lo tanto $\Delta: \mathcal{E}^{\prime m} \widehat{\otimes}_{\pi} \mathcal{E} \rightarrow \mathcal{D}^{\prime m}$ es continuo.

De la misma manera se obtiene [11] que $\Delta$ es continuo en los espacios: $\mathcal{E}^{\prime m}\left(\mathbb{R}^{n}\right) \widehat{\otimes}_{\pi}$ $\mathcal{E}\left(\mathbb{R}^{n}\right), \mathcal{S}^{\prime m}\left(\mathbb{R}^{n}\right) \widehat{\otimes}_{\pi} \mathcal{O}_{M}\left(\mathbb{R}^{n}\right), \mathcal{O}_{C}^{\prime m}\left(\mathbb{R}^{n}\right) \widehat{\otimes}_{\pi} \mathcal{O}_{C}\left(\mathbb{R}^{n}\right), \mathcal{P}^{\prime m}\left(\mathbb{R}^{n}\right) \widehat{\otimes}_{\pi} \mathcal{P}\left(\mathbb{R}^{n}\right), \mathcal{K}^{\prime m}\left(\mathbb{R}^{n}\right) \widehat{\otimes}_{\pi}$ $\mathcal{K}\left(\mathbb{R}^{n}\right)$.

\section{$4 \quad$ La expansión en $\mathcal{A}^{\prime m}\left(\mathbb{R}^{n}\right) \widehat{\otimes}_{\pi} \mathcal{A}\left(\mathbb{R}^{n}\right)$}

La introducción de los espacios donde el operador $\Delta$ es lineal y continuo, nos va a permitir obtener la expansión de $f(\lambda \mathbf{x}, \mathbf{x})$, cuando $\lambda \rightarrow+\infty$.

\section{Teorema 4.1:}

Sea $\mathcal{A}$ uno de los espacios $\mathcal{E}, \mathcal{O}_{C}, \mathcal{K}$ o $\mathcal{P}$. Sea $f \in \mathcal{A}^{\prime m}\left(\mathbb{R}^{n}\right) \widehat{\otimes}_{\pi} \mathcal{A}\left(\mathbb{R}^{n}\right)$ para algún $m \in \mathbb{I N}$. Entonces

$$
f(\lambda \mathbf{x}, \mathbf{x})=\sum_{\mathbf{k} \in \mathbb{N}^{n}} \sum_{\mathbf{j} \leq \mathbf{k}} \frac{(-1)^{|\mathbf{k}-\mathbf{j}|} \mu_{\mathbf{k}}^{\mathbf{j}} \mathbf{D}^{\mathbf{k}-\mathbf{j}} \delta(\mathbf{x})}{\mathbf{j} !(\mathbf{k}-\mathbf{j}) ! \lambda^{|\mathbf{k}|+n}}+O\left(\frac{1}{\lambda^{N+n+1}}\right), \text { cuando } \lambda \rightarrow+\infty,
$$

donde $\mu_{\mathbf{k}}(\mathbf{y})=\left\langle f(\mathbf{x}, \mathbf{y}), \mathbf{x}^{\mathbf{k}}\right\rangle, \mathbf{k} \in I^{n}$ son los momentos parciales de $f$ y $\mu_{\mathbf{k}}^{\mathbf{j}}$ están dados por

$$
\mu_{\mathbf{k}}^{\mathbf{j}}=\mathbf{D}^{\mathbf{j}} \mu_{\mathbf{k}}(\mathbf{0}),
$$

PRUEBa: Si $f \in \mathcal{A}^{\prime m}\left(\mathbb{R}^{n}\right) \widehat{\otimes}_{\pi} \mathcal{A}\left(\mathbb{R}^{n}\right)$, entonces la expansión

$$
f(\lambda \mathbf{x}, \mathbf{y})=\sum_{|\mathbf{k}|=0}^{N} \frac{(-1)^{|\mathbf{k}|} \mu_{\mathbf{k}}(\mathbf{y}) \mathbf{D}^{\mathbf{k}} \delta(\mathbf{x})}{\mathbf{k} ! \lambda^{|\mathbf{k}|+n}}+O\left(\frac{1}{\lambda^{N+n+1}}\right)
$$

cuando $\lambda \rightarrow \infty$, es valida en el espacio $\mathcal{A}^{\prime p}\left(\mathbb{R}^{n}\right) \widehat{\otimes}_{\pi} \mathcal{A}\left(\mathbb{R}^{n}\right)$ para algún $p \geq \max \{m, N\}$. Aplicando el operador $\Delta$ a (4.3) y teniendo en cuenta que

$$
\mu(\mathbf{x}) \mathbf{D}^{\mathbf{k}} \delta(\mathbf{x})=\sum_{\mathbf{j} \leq \mathbf{k}}(-1)^{|\mathbf{j}|} \frac{\mathbf{k} !}{\mathbf{j} !(\mathbf{k}-\mathbf{j}) !} \mathbf{D}^{\mathbf{j}} \mu(\mathbf{0}) \mathbf{D}^{\mathbf{k}-\mathbf{j}} \delta(\mathbf{x}),
$$


se obtiene

$$
f(\lambda \mathbf{x}, \mathbf{x}) \sim \sum_{\mathbf{k} \in N^{n}} \sum_{\mathbf{j} \leq \mathbf{k}} \frac{(-1)^{|\mathbf{k}-\mathbf{j}|} \mu_{\mathbf{k}}^{\mathbf{j}} \mathbf{D}^{\mathbf{k}-\mathbf{j}} \delta(\mathbf{x})}{\mathbf{j} !(\mathbf{k}-\mathbf{j}) ! \lambda^{|\mathbf{k}|+n}}, \text { cuando } \lambda \rightarrow+\infty
$$

Ejemplo. Como ejemplo de la aplicación de este teorema consideremos el funcional

$$
\Psi(\lambda):=\int_{\mathbb{R}^{2}} e^{-\lambda\left(x_{1}^{2}+x_{2}^{2}\right)}\left(x_{1}^{2}+x_{2}^{2}\right) \phi\left(x_{1}, x_{2}\right) d x_{1} d x_{2}, \quad \phi \in \mathcal{P} .
$$

Entonces se tiene que $\Psi(\lambda)=\langle f(\lambda \mathbf{x}, \mathbf{x}), \phi(\mathbf{x})\rangle$, donde $f(\mathbf{x}, \mathbf{y})=e^{-|\mathbf{x}|^{2}}|y|^{2}$; entonces $f \in \mathcal{P}^{\prime m} \widehat{\otimes}_{\pi} \mathcal{P}$ y por el teorema 4.1 sabemos que

$$
f(\lambda \mathbf{x}, \mathbf{x}) \sim \sum_{\mathbf{k} \in \mathbb{I} N^{n}} \sum_{\mathbf{j} \leq \mathbf{k}} \frac{(-1)^{|\mathbf{k}-\mathbf{j}|} \mu_{\mathbf{k}}^{\mathbf{j}} \mathbf{D}^{\mathbf{k}-\mathbf{j}} \delta(\mathbf{x})}{\mathbf{j} !(\mathbf{k}-\mathbf{j}) ! \lambda^{|\mathbf{k}|+n}}, \text { cuando } \lambda \rightarrow+\infty,
$$

donde los correspondientes momentos (4.2), están dados por

$$
\begin{aligned}
\mu_{\mathbf{k}}^{\mathbf{j}} & =\mathbf{D}^{\mathbf{j}}\left(\mu_{\mathbf{k}}(\mathbf{y})\right)_{\mathbf{y}=0} \\
& =\mathbf{D}^{\mathbf{j}}\left(|\mathbf{y}|^{2} \Gamma\left(\frac{2 k_{1}+1}{2}\right) \Gamma\left(\frac{2 k_{2}+1}{2}\right)\right)_{\mathbf{y}=0},
\end{aligned}
$$

o bien

$$
\mu_{\left(k_{1}, k_{2}\right)}^{\left(j_{1}, j_{2}\right)}=\frac{\partial^{j_{1}}}{\partial y_{1}^{j_{1}}} \frac{\partial^{j_{2}}}{\partial y_{2}^{j_{2}}}\left(\left(y_{1}^{2}+y_{2}^{2}\right) \Gamma\left(\frac{2 k_{1}+1}{2}\right) \Gamma\left(\frac{2 k_{2}+1}{2}\right)\right)_{(0,0)},
$$

si $\mathbf{k}=\left(k_{1}, k_{2}\right), \mathbf{j}=\left(j_{1}, j_{2}\right)$.

Desarrollando los primeros términos $N=3$ y usando (4.4), encontramos que

$$
\begin{aligned}
f(\lambda \mathbf{x}, \mathbf{x}) & \sim \frac{2 \Gamma\left(\frac{5}{2}\right) \Gamma\left(\frac{1}{2}\right)}{\lambda^{4}} \delta(\mathbf{x})-\frac{\Gamma\left(\frac{5}{2}\right) \Gamma\left(\frac{3}{2}\right)}{\lambda^{5}}\left[\frac{\partial}{\partial x_{2}} \delta(\mathbf{x})+\frac{\partial}{\partial x_{1}} \delta(\mathbf{x})\right] \\
& -\frac{\Gamma\left(\frac{7}{2}\right) \Gamma\left(\frac{1}{2}\right)}{\lambda^{5}}\left[\frac{\partial}{\partial x_{1}} \delta(\mathbf{x})+\frac{\partial}{\partial x_{2}} \delta(\mathbf{x})\right]
\end{aligned}
$$

por lo tanto

$$
\begin{aligned}
\Psi(\lambda) & =\frac{2 \Gamma\left(\frac{5}{2}\right) \Gamma\left(\frac{1}{2}\right)}{\lambda^{4}} \phi(0,0)+\frac{\Gamma\left(\frac{5}{2}\right) \Gamma\left(\frac{3}{2}\right)}{\lambda^{5}}\left[\frac{\partial}{\partial x_{2}} \phi\left(x_{1}, 0\right)+\frac{\partial}{\partial x_{1}} \phi\left(0, x_{2}\right)\right] \\
& +\frac{\Gamma\left(\frac{7}{2}\right) \Gamma\left(\frac{1}{2}\right)}{\lambda^{5}}\left[\frac{\partial}{\partial x_{1}} \phi\left(0, x_{2}\right)+\frac{\partial}{\partial x_{2}} \phi\left(x_{1}, 0\right)\right]+O\left(\lambda^{-6}\right), \quad \lambda \rightarrow+\infty .
\end{aligned}
$$

Ejemplo. Si $\phi \in \mathcal{S}\left(\mathbb{R}^{n}\right)$, entonces su transformada de Fourier está dada por

$$
\widehat{\phi}(u)=\mathcal{F}\{\phi(\mathbf{x}) ; \mathbf{u}\}=\int_{\mathbb{R}^{n}} \phi(\mathbf{x}) e^{i \mathbf{x} \cdot \mathbf{u}} d \mathbf{x} .
$$


Puesto que la transformada de Fourier es un isomorfismo [3] de $\mathcal{S}\left(\mathbb{R}^{n}\right)$ en $\mathcal{S}\left(\mathbb{R}^{n}\right)$. Teniendo en cuenta que [9]

$$
\mathcal{S}\left(\mathbb{R}^{2 n}\right) \hookrightarrow \mathcal{A}^{\prime m}\left(\mathbb{R}^{n}\right) \widehat{\otimes}_{\pi} \mathcal{A}\left(\mathbb{R}^{n}\right),
$$

por ejemplo para $\mathcal{A}=\mathcal{O}_{C}$. Se sigue que la expansión (4.1) es valida en $\mathcal{S}\left(\mathbb{R}^{2 n}\right)$ y por lo tanto $\widehat{\phi}$, admite la expansión

$$
\widehat{\phi}(\lambda \mathbf{u}, \mathbf{u}) \sim \sum_{\mathbf{k} \in I N^{n}} \sum_{\mathbf{j} \leq \mathbf{k}} \frac{(-1)^{|\mathbf{k}-\mathbf{j}|} \mu_{\mathbf{k}}^{\mathbf{j}} \mathbf{D}^{\mathbf{k}-\mathbf{j}} \delta(\mathbf{u})}{\mathbf{j} !(\mathbf{k}-\mathbf{j}) ! \lambda|\mathbf{k}|+n}, \text { cuando } \lambda \rightarrow+\infty .
$$

Ejemplo. Consideremos la siguiente función generalizada

$$
\Phi(\epsilon):=\sum_{n=1}^{\infty} n^{-p} \sin \left(\frac{\epsilon}{n^{2}}\right) \delta\left(x-\frac{\epsilon}{n}\right), \quad p>1, \epsilon \rightarrow 0^{+} .
$$

Es fácil ver que $\sum_{n=1}^{\infty}\left|a_{n}^{(q)}(y)\right|$ converge para todo $q=0,1, \ldots$, donde $a_{n}(y)=n^{-p} \sin \left(\frac{y}{n}\right)$. Además el núcleo $\sum_{n=1}^{\infty} n^{-p} \sin \left(\frac{y}{n}\right) \delta\left(x-\frac{1}{n}\right) \in \mathcal{E}^{\prime m}\left(\mathbb{R}^{n}\right) \widehat{\otimes}_{\pi} \mathcal{E}\left(\mathbb{R}^{n}\right), m=0$.

Por el teorema 4.1, obtenemos

$$
\Phi(\epsilon) \sim \sum_{k=0}^{\infty} \sum_{j=0}^{k} \frac{(-1)^{k-j} \mu_{k}^{j} \mathbf{D}^{k-j} \delta(x)}{j !(k-j) !} \epsilon^{k}, \text { cuando } \epsilon \rightarrow 0^{+}
$$

donde

$$
\mu_{k}^{j}=\sum_{n=1}^{\infty} \frac{a_{n}^{(j)}(0)}{n^{k}}
$$

Teniendo en cuenta que

$$
a_{n}^{(q)}(0)=\left\{\begin{array}{lll}
0 & \text { si } q=2 j, & j=1,2, \ldots \\
\frac{(-1)^{q+1}}{n^{p+q}} & \text { si } q=2 j-1, & j=1,2, \ldots
\end{array},\right.
$$

obtenemos que

$$
\Phi(\epsilon) \sim \sum_{k=1}^{\infty} \sum_{m=1}^{\left\lfloor\frac{1+k}{2}\right\rfloor} \frac{(-1)^{k-m} \zeta(p+2 m+k-1) \delta^{(k+1-2 m)}(x)}{(2 m-1) !(k+1-2 m) !} \epsilon^{k}, \text { cuando } \epsilon \rightarrow 0^{+},
$$

donde $\zeta$ es la función zeta de Riemann y $\lfloor$ 」 significa parte entera.

En particular, si $p+2 m+k-1 \in \mathbb{Z}$ y es par, podemos obtener los valores de $\zeta$ a partir de [3]

$$
\zeta(2 k)=\frac{(-1)^{k-1} B_{2 k}(2 \pi)^{2 k}}{2(2 k) !},
$$

donde los $B_{2 k}$ son los coeficientes de Bernoulli. 
Si $\phi \in \mathcal{E}(\mathbb{R})$ se tiene

$$
\sum_{n=1}^{\infty} n^{-p} \sin \left(\frac{\epsilon}{n^{2}}\right) \phi\left(\frac{\epsilon}{n}\right) \sim \sum_{k=1}^{\infty} \sum_{m=1}^{\left\lfloor\frac{1+k}{2}\right\rfloor} \frac{(-1)^{1-3 m} \zeta(p+2 m+k-1) \phi^{(k+1-2 m)}(0)}{(2 m-1) !(k+1-2 m) !} \epsilon^{k},
$$

cuando $\epsilon \rightarrow 0^{+}$.

En particular si $\phi \equiv 1$ se obtiene

$$
\sum_{n=1}^{\infty} n^{-p} \sin \left(\frac{\epsilon}{n^{2}}\right) \sim \sum_{j=1}^{\infty} \frac{(-1)^{j+1} \zeta(p+4 j-2)}{(2 j-1) !} \epsilon^{2 j-1}, \text { cuando } \epsilon \rightarrow 0^{+}
$$

Si $p$ es par, entonces del teorema 4.1 y usando (4.5) se obtiene

$$
\sum_{n=1}^{\infty} n^{-p} \sin \left(\frac{\epsilon}{n^{2}}\right)=\sum_{j=1}^{N} \frac{(-1)^{\frac{p}{2}+3 j-1}(2 \pi)^{p+4 j-2} B_{p+4 j-2}}{2(2 j-1) !(p+4 j-2) !} \epsilon^{2 j-1}+O\left(\epsilon^{N+2}\right),
$$

cuando $\epsilon \rightarrow 0^{+}$.

La expansión (4.1) no es válida [3] en $\mathcal{S}^{\prime}\left(\mathbb{R}^{n}\right)$, sin embargo si se introducen algunas condiciones se puede obetener la siguiente expansión.

Teorema 4.2: Sea $f \in \mathcal{S}^{\prime m}\left(\mathbb{R}^{n}\right) \widehat{\otimes}_{\pi} \mathcal{O}_{M}\left(\mathbb{R}^{n}\right)$ con sop $(f) \subseteq B \times \mathbb{R}$ donde $B=$ $\left[A_{1}, \infty\left[\times \ldots \times\left[A_{n}, \infty\left[\right.\right.\right.\right.$, para algunas constantes $A_{k}>0, k=1,2, \ldots, n$

Suponga

$$
\mathbf{D}_{\mathbf{y}}^{\mathbf{j}} f(\mathbf{x}, \mathbf{y})=\sum_{i=1}^{n} \mathbf{x}^{\mathbf{a}_{i}} \mathbf{D}^{\mathbf{j}} \psi_{i}(\mathbf{y})+O\left(\mathbf{x}^{\mathbf{a}_{n+1}} \mathbf{D}^{\mathbf{j}} \psi_{n+1}(\mathbf{y})\right),
$$

cuando $\mathbf{x} \rightarrow \infty$, para $0 \leq|\mathbf{j}| \leq N+1$, donde $\mathbf{a}_{1}>\mathbf{a}_{2}>\ldots>\mathbf{a}_{n+1} y-(N+2)<\left|\mathbf{a}_{n+1}\right|<$ $-(N+1)$.

Entonces

$$
f(\lambda \mathbf{x}, \mathbf{x})=\sum_{i=1}^{n} \operatorname{Pf}\left((\lambda \mathbf{x})^{\mathbf{a}_{i}} H(\lambda \mathbf{x})\right) \psi_{i}(\mathbf{x})+\sum_{\mathbf{k} \in N^{n}} \sum_{\mathbf{j} \leq \mathbf{k}} \frac{(-1)^{|\mathbf{k}-\mathbf{j}|} \mu_{\mathbf{k}}^{\mathbf{j}} \mathbf{D}^{\mathbf{k}-\mathbf{j}} \delta(\mathbf{x})}{(\mathbf{k}-\mathbf{j}) ! \mathbf{j} ! \lambda^{|\mathbf{k}|+n}}+O\left(\lambda^{\left|\mathbf{a}_{n+1}\right|}\right),
$$

cuando $\lambda \rightarrow+\infty$ en el espacio $\mathcal{S}^{\prime}$, donde $\operatorname{Pf}\left(\mathbf{x}^{\mathbf{a}} H(\mathbf{x})\right)$ es la distribución dada por la parte finita de Hadamard

$$
\left\langle P f\left(\mathbf{x}^{\mathbf{a}} H(\mathbf{x})\right), \phi(\mathbf{x})\right\rangle=F \cdot p \int_{\mathbb{R}^{n}} \mathbf{x}^{\mathbf{a}} \phi(\mathbf{x}) d \mathbf{x},
$$

$y$ donde $\mu_{\mathbf{k}}^{\mathbf{j}}$ son los momentos generalizados

$$
\mu_{\mathbf{k}}^{\mathbf{j}}=\mathbf{D}^{\mathbf{j}}\left\langle f(\mathbf{x}, \mathbf{y})-\sum_{i=1}^{n} \operatorname{Pf}\left(\mathbf{x}^{\mathbf{a}_{i}} H(\mathbf{x})\right) \psi_{i}(\mathbf{y}), \mathbf{x}^{\mathbf{k}}\right\rangle_{\mathbf{y}=0}
$$




\section{Referencias}

[1] Brüning, J.; Seeley, R. (1958) "Regular Singular Asymptotics", Advances in Mathematics 58: 133-148.

[2] Brüning, J.; Seeley, R. (1991) "The Expansion of the Resolvent", Journal of Functional Analysis 95: 255-290.

[3] Estrada, R.; Kanwal, R.P. (1993) Asymptotic Analysis: a Distributional Approach. Birkäuser, Boston.

[4] Estrada, R.; Kanwal, R. P. (1990) "A distributional theory for asymptotic expansions", Proc. R. Soc.Lond. A 428: 399-430.

[5] Horváth, J. (1966) Topological Vector Spaces and Distributions, Vol. 1. AddisonWesley, Reading Mass.

[6] Sellier, A. "Asymptotic expansions of a class of integrals", preprint.

[7] Sellier, A. "Hadamard's finite part concept in dimension $n \geq 2$. Distributional definition. Regularization forms and distributional derivatives", to appear in Proc. Roy. Soc. London A.

[8] Treves, F. (1967) Topological Vector Spaces, Distributions and Kernels. Academic Press, New York.

[9] Ulate, C.M.; Estrada, R. "Expansion of distributional kernels of the type $f(\lambda x, x)$, as $\lambda \rightarrow \infty "$, por publicarse.

[10] Ulate, C.M. (1994) "La expansión asintótica de momentos para núcleos de la forma $f(\lambda x, x)$, cuando $\lambda \rightarrow+\infty$ si $f \in \mathcal{S}_{x}^{\prime m}(\mathbb{R}) \widehat{\otimes}_{\pi} \mathcal{O}_{M y}(\mathbb{R})$ ". En: Memoria II Encuentro Centroamericano de Investigadores en Matemáticas, I Parte, G. Mora (editor). San Ramón, Sede de Occidente U.C.R., pp. 207-215.

[11] Ulate, C. M. Tesis de Grado para optar al título de Licenciado en Matemática, Universidad de Costa Rica, en preparación. 